\title{
Criopreservação do Sêmen de Tilápia-Nilótica Oreochromis niloticus, var. Chitralada: Crioprotetores, Soluções Ativadoras e Refrigerador Criogênico ${ }^{1}$
}

\author{
Hugo Pereira Godinho², Vanessa Márcia da Cunha Amorim³, Marco Túlio Diniz Peixoto ${ }^{4}$
}

\begin{abstract}
RESUMO - Realizaram-se experimentos relativos a crioprotetores, diluentes e soluções ativadoras pós-descongelamento com o sêmen de tilápia-nilótica Oreochromis niloticus, var. Chitralada. Utilizaram-se soluções crioprotetoras contendo DMSO ou metanol, em várias concentrações. O sêmen diluído na solução crioprotetora foi envazado em palhetas de $0,5 \mathrm{~mL}$, congelado em botijão de vapor de nitrogênio líquido e, então, armazenado em nitrogênio líquido. A motilidade espermática do sêmen descongelado foi avaliada em soluções ativadoras de $\mathrm{NaHCO}_{3} 119 \mathrm{mM}, \mathrm{NaCl} 25 \mathrm{mM}$ e água destilada. As taxas de motilidade espermática pós-descongelamento obtidas com sêmen criopreservado com metanol (concentração final=7,5-12 \%) foram de 31-46\% e quando o crioprotetor utilizado foi o DMSO (concentração final = 5-8\%), de 20-35\%. Em ambas as condições, o sêmen foi ativado com solução de $\mathrm{NaHCO}_{3} 119 \mathrm{mM}$. A diluição do sêmen em diferentes proporções (1:1 a 1:4; sêmen: diluente) não produziu diferenças significativas nas taxas de motilidade espermática pós-descongelamento.
\end{abstract}

Palavras-chave: criopreservação do sêmen, crioprotetores, taxa de diluição do sêmen, taxa de motilidade espermática, tilápia-nilótica

\section{Cryopreservation of the Semen of Nile Tilapia Oreochromis niloticus, var. Chitralada: Cryoprotectants, Spermatozoa Activating Solutions and Cryogenic Refrigerator}

\begin{abstract}
Experiments were conducted concerning cryoprotectants, extenders, and post thawing activating solutions for the semen of Nile tilapia Oreochromis niloticus, var. Chitralada. The extender solutions contained DMSO or methanol in various concentrations. The semen was previously diluted in the extender, drawn into $0.5 \mathrm{~mL}$ straws, frozen in the cryogenic shipper and then stored in liquid nitrogen. Motility of the thawed sperm was evaluated with $\mathrm{NaHCO}_{3} 119 \mathrm{mM}, \mathrm{NaCl} 25 \mathrm{mM}$ and distilled water. Motility rate of thawed sperm, cryopreserved with methanol (final concentration $=7.5-12 \%$ ) and activated with $\mathrm{NaHCO}_{3} 119 \mathrm{mM}$, was $31-46 \%$. When DMSO replaced methanol, the motility of frozen/thawed sperm activated, also with $\mathrm{NaHCO}_{3} 119 \mathrm{mM}$, was $20-35 \%$. Semen:extender dilutions of $1: 1$ to $1: 4$ did not show significant effects on the post-thawing sperm motility rate.
\end{abstract}

Key Words: sperm cryopreservation, cryoprotectants, semen dilution rate, sperm motility rate, Nile tilapia

\section{Introdução}

A reprodução e a genética seletiva constituem temas prioritários de pesquisa voltados para o incremento da produção comercial de tilápias (Engle, 1997). Nesse contexto, a criopreservação de espermatozóides de tilápias assume papel relevante para a aqüicultura e para a conservação de seus recursos genéticos (Rana \& McAndrew, 1989). Várias atividades podem ser otimizadas com o uso de sêmen congelado, incluindo sua obtenção em tempos e locais diferentes e utilização em programas de melhoramento genético. Não obstante os progressos obtidos na criopreservação de sêmen de peixes, os resultados práticos obtidos ainda estão sujeitos à grandes variações, resultando na necessidade do desenvolvimento de tecnologias adequadas visando a espécie e a interespecificidade (Piironen, 1993). Bancos de sêmen têm sido montados utilizando-se técnica simples e facilmente aplicável em condições de campo. Essa técnica, inicialmente desenvolvida para programas de conservação genética do salmãodo-pacífico (Harvey, 1998), utiliza um refrigerador criogênico especial, o botijão de vapor de nitrogênio líquido, e tem sido empregada para criopreservação do sêmen de várias espécies nativas sul-americanas (Harvey, 2000). As taxas de congelamento desse refrigerador, situadas entre -20 e $-40^{\circ} \mathrm{C} / \mathrm{min}$

\footnotetext{
1 Parte da dissertação de Mestrado apresentada por VMCA ao Programa de Pós-graduação em Zoologia de Vertebrados, Pontifícia Universidade Católica de Minas Gerais, Belo Horizonte.

2 Professor-Adjunto, Programa de Pós-graduação em Zoologia de Vertebrados, Pontifícia Universidade Católica de Minas Gerais, Av. Dom José Gaspar, 500, Belo Horizonte, MG, 30535-610. E-mail: hgodinho@pucminas.br

${ }^{3}$ Mestre em Zoologia de Vertebrados, Programa de Pós-graduação em Zoologia de Vertebrados, Pontifícia Universidade Católica de Minas Gerais, Av. Dom José Gaspar, 500, Belo Horizonte, MG, 30535-610.

${ }^{4}$ Médico-Veterinário, Multi Gene Aqüicultura, Fazenda Água Fria, C.P. 46, Esmeraldas, MG, 35740-000. E-mail: mtdp@brhs.com.br
} 
(Mongkonpunya et al., 2000), são consideradas adequadas para a criopreservação do sêmen de peixes (Harvey \& Carolsfeld, 1993). Além de ser de fácil transporte por conter apenas vapor de nitrogênio líquido, ele mantém a temperatura de criopreservação por até três semanas sem necessidade de reabastecimento o que o torna bastante prático para trabalhos de campo (Wayman \& Tiersch, 2000).

As tilápias constituem um dos mais importantes grupos de peixes em termos de produção mundial perdendo apenas para carpas e salmonídeos. Embora a produção brasileira de tilápias venha crescendo, ela ainda é inexpressiva se comparada à de outros países (FAO, 1997). A tilápia-nilótica (Oreochromis niloticus) var. Chitralada, de origem tailandesa, foi recentemente introduzida no Brasil e seu cultivo temse difundido rapidamente em várias regiões do país (Boscolo et al., 2001).

Em vista de os dados obtidos na literatura serem conflitantes em relação à criopreservação do sêmen de tilápias, especialmente quanto às taxas de diluição do sêmen e soluções ativadoras (Rana \& McAndrew, 1989), avaliaram-se, neste trabalho, metodologias de criopreservação a serem utilizadas na montagem de bancos de sêmen de tilápia-nilótica, var. Chitralada. Assim, foram avaliadas: a) toxicidade de crioprotetores, b) diferentes taxas de diluição do sêmen e c) soluções ativadoras da motilidade espermática pós-descongelamento. Além disto, o botijão de vapor de nitrogênio líquido foi, pela primeira vez, utilizado como o recipiente criogênico na criopreservação do sêmen dessa espécie.

\section{Material e Métodos}

Os peixes e coleta do sêmen

Utilizou-se sêmen de tilápias-nilóticas (Oreochromis niloticus), variedade Chitralada, pertencentes à uma piscicultura comercial. Os peixes foram separados por sexo e mantidos em tanque de argamassa armada com $3,6 \mathrm{~m}^{3}$ de capacidade e com sistemas de filtragem e recirculação da água. Diariamente, os animais foram alimentados com ração extrusada contendo $28 \%$ de proteína bruta, fornecida em $2 \%$ da biomassa, duas vezes ao dia. Os experimentos foram realizados na estação de piscicultura comercial de origem dos peixes, no período de setembro a dezembro de 2001.

Os doadores de sêmen foram selecionados de acordo com a coloração avermelhada da papila urogenital e que eliminavam algumas gotas de sêmen por leve compressão manual da cavidade celômica. Após a seleção, os machos foram pesados (g) e medidos $(\mathrm{cm})$. Durante a coleta de sêmen, utilizaram-se toalhas umedecidas para imobilizar os peixes e vendar seus olhos. A papila urogenital foi seca com toalha de papel para reduzir o risco de possível contaminação com água, fezes ou urina. $\mathrm{O}$ sêmen foi recolhido em seringas graduadas (capacidade 1,0 mL) e anotado o volume.

\section{Teste de contaminação do sêmen}

Imediatamente após a coleta, uma gota de sêmen fresco de cada peixe foi colocada no microscópio de luz, previamente focalizado em 400x, para exame da motilidade espermática. Tendo em vista que os espermatozóides no sêmen fresco, isento de contaminação, são imóveis, considerou-se adequado para os experimentos o sêmen que exibiu taxas de motilidade espermática não superiores a $1 \%$.

Experimento 1 - Toxicidade de crioprotetores para o sêmen fresco

Para realização deste teste, utilizaram-se os seguintes crioprotetores: metanol, dimetilsulfóxido (DMSO), etilenoglicol, dimetilacetamida (DMA) e propanodiol. A cada $10 \mu \mathrm{L}$ de solução de 5, 10 e 15\% de cada crioprotetor diluiu-se $1 \mu \mathrm{L}$ de sêmen, tendo registrado, ao microscópio de luz, a taxa de motilidade espermática e o tempo de latência. A taxa de motilidade espermática foi obtida por meio da avaliação subjetiva feita, imediatamente, após a diluição do sêmen. O tempo decorrido entre a mistura de sêmen com o crioprotetor e o início da motilidade espermática foi considerado como tempo de latência.

Experimento 2 - Efeito sobre a motilidade espermática pós-descongelamento de sêmen congelado com diferentes crioprotetores

Em todos os experimentos de criopreservação, foram utilizadas apenas amostras de sêmens não contaminados e com motilidade espermática acima de $80 \%$. O processo de criopreservação baseou-se no protocolo de Harvey (2000), utilizando-se o botijão de vapor de nitrogênio líquido (Taylor-Wharton, modelo CP 65).

Para este experimento, três "pools" de sêmen coletado de, respectivamente, 4 , 4 e 3 peixes, foram misturados à soluções diluentes na proporção de 1:9 (sêmen:diluente) segundo Harvey \& Kelley (1988). As soluções diluentes continham 5\% de glicose, $75 \%$ de água destilada e 10\% de DMSO ou de etilenoglicol ou de metanol. Aos diluentes contendo DMSO ou etilenoglicol, adicionaram-se $10 \%$ de gema fresca de ovo de galinha, ao passo que naquele contendo metanol 
adicionaram-se $10 \%$ de leite em pó desnatado, de acordo com Harvey (1983). O sêmen diluído foi envasado em palhetas plásticas com capacidade de $0,5 \mathrm{~mL}$ (IMV, França) e dispostos nos compartimentos superior e inferior de suportes de alumínio. Em seguida, esses suportes foram imediatamente colocados no botijão de vapor de nitrogênio. Decorridas 3 horas, os suportes foram transferidos para botijão de nitrogênio líquido onde permaneceram até sua utilização.

A fim de se estimar a taxa de motilidade espermática do sêmen descongelado, cada palheta foi retirada do botijão de nitrogênio líquido, colocada em banho-maria a $30^{\circ} \mathrm{C}$, durante $\sim 6 \mathrm{~s}$ e seu conteúdo, ainda pastoso, foi despejado em placas de Petri à temperatura ambiente $\left(\sim 22-25^{\circ} \mathrm{C}\right)$. Alíquotas de $\sim 2 \mu \mathrm{L}$ do sêmen descongelado foram colocadas em lâmina histológica à qual juntaram-se $10 \mu \mathrm{L}$ de uma das soluções ativadoras da motilidade espermática a serem testadas: $\mathrm{NaHCO}_{3} 119 \mathrm{mM}, \mathrm{NaCl} 25 \mathrm{mM}$ e água destilada. Após rápida homogeneização, as taxas de motilidade espermática (\%) foram estimadas através de análise subjetiva em microscópio de luz com 400x de aumento.

Experimento 3 - Efeito da diluição do sêmen na motilidade espermática pós-descongelamento

Utilizou-se sêmen de três peixes e de um "pool", para testar dois diluentes contendo diferentes concentrações de crioprotetores. O primeiro continha $10 \%$ de DMSO, $10 \%$ de gema de ovo , $5 \%$ de glicose e água destilada (q.s.p. 100\%). O segundo diluente continha $15 \%$ de metanol, $15 \%$ de leite em pó, $5 \%$ de glicose e água destilada (q.s.p. 100\%). As taxas de diluição do sêmen foram: 1:1, 1:2, 1:3 e 1:4 (sêmen:diluente, v/v), o que correspondeu às taxas de concentração final dos crioprotetores de, respectivamente, 7,$5 ; 10,0 ; 11,3$ e $12,0 \%$ para o metanol e $5 ; 6,7$; 7,5 e 8,0\% para DMSO. O envazamento do sêmen diluído e seu congelamento foram realizados tal como descrito para o Exp. 2. A estimativa das taxas de motilidade espermática pós-descongelamento seguiu o protocolo do Exp. 1. A duração da motilidade espermática foi registrada até quando restaram $\sim 20 \%$ de espermatozóides móveis. A ativação da motilidade espermática foi conduzida tal como indicada no Exp. 2, utilizando-se $\mathrm{NaHCO}_{3} 119 \mathrm{mM}$ como solução ativadora.

\section{Análises estatísticas}

Para análise dos testes de toxicidade e de taxa de motilidade espermática pós-descongelamento utilizou-se o teste não-paramétrico de Kruskal-Wallis, nível de significância $=0,05 \%$. As variações entre durações da motilidade espermática e entre os tempos de latência foram analisadas por meio de ANOVA (nível de significância $=0,05 \%$ ).

\section{Resultados e Discussão}

Várias técnicas de coleta de sêmen de peixes estão disponíveis na literatura (Yao et al., 2000). No presente trabalho, a coleta de sêmen com seringas de $1 \mathrm{~mL}$ foi facilitada em razão da conformação anatômica da papila urogenital, embora tenham sido descartadas cerca de $7 \%$ das amostras (6 em 83 coletas) com suspeita de contaminação. A suspeita de contaminação, possivelmente, por urina decorreu do fato dessas amostras terem apresentado ao exame microscópico, realizado a fresco, taxas de motilidade espermática superiores a $1 \%$.

Os resultados dos testes de toxicidade dos crioprotetores do experimento 1 estão na Tabela 1 . Dentre os crioprotetores utilizados, as diferentes concentrações de metanol, etilenoglicol e propanodiol não influenciaram significativamente nas taxas de motilidade espermática. Todavia, concentrações de $15 \%$ de DMSO e DMA induziram taxas de motilidade espermática significativamente mais baixas do que as de $5 \%$ e $10 \%$, especialmente o DMA. A motilidade espermática das amostras de sêmen diluídas em metanol e DMA foi imediatamente iniciada (tempo de latência $=0 \mathrm{~s}$ ). Entretanto, a motilidade espermática das amostras de sêmen diluídas em DMSO, etilenoglicol e propanodiol levou um tempo para ter início. Em soluções de DMSO e de etilenoglicol, o tempo de latência aumentou significativamente nas concentrações mais altas (Tabela 1).

Efeitos tóxicos de crioprotetores no sêmen de peixes têm sido observados especialmente quando utilizados em altas concentrações (Leung, 1991). Por essa razão, os testes de toxicidade deste trabalho envolveram concentrações comumente utilizadas em criopreservação. DMSO tem sido utilizado com sucesso em diferentes espécies de peixes, tal como em Macrozoarces americanus (Yao et al., 2000). DMA é pouco utilizado, embora tenha sido empregado com sucesso no bagre-africano Clarias gariepinus (Horváth \& Urbányi, 2000). O metanol é considerado o mais adequado para a criopreservação de sêmen de tilápias (Harvey, 1983; Chao et al., 1987; Harvey \& Kelley, 1988; Rana \& McAndrew, 1989). A eficiência crioprotetora aumenta, quando dois crioprotetores 
Tabela 1 - Toxicidade de crioprotetores, avaliada com base na taxa de motilidade espermática e tempo de latência (média $\pm \mathrm{dp}, \mathrm{n}=6$ ) para o sêmen fresco de tilápia-nilótica (Oreochromis niloticus), var. Chitralada

Table 1 - Cryoprotectant toxicity, evaluated through motility rate and latency time (mean $\pm s . d$., $n=6$ ) of fresh sperm of the Nile tilapia (Oreochromis niloticus), var. Chitralada

\begin{tabular}{|c|c|c|c|}
\hline & $\begin{array}{c}\text { Crioprotetor } \\
\text { (concentração, \%) } \\
\text { Cryoprotectant } \\
\text { (concentration) }\end{array}$ & $\begin{array}{c}\text { Taxa de motilidade } \\
\text { Motility rate } \\
\text { (\%) }\end{array}$ & $\begin{array}{c}\text { Tempo de latência } \\
\text { Latency time } \\
\text { (s) }\end{array}$ \\
\hline \multicolumn{4}{|l|}{ Metanol } \\
\hline \multicolumn{4}{|l|}{ Methanol } \\
\hline & 5 & $90,0 \pm 8,9^{a b}$ & $0,0 \pm 0,0^{\mathrm{a}}$ \\
\hline & 10 & $92,5 \pm 6,1^{\mathrm{a}}$ & $0,0 \pm 0,0^{\mathrm{a}}$ \\
\hline & 15 & $83,3 \pm 8,2^{b}$ & $0,0 \pm 0,0^{\mathrm{a}}$ \\
\hline DMSO & 5 & $93,3 \pm 5,2^{\mathrm{a}}$ & $0.0 \pm 0.0^{\mathrm{a}}$ \\
\hline & 10 & $92,5 \pm 9,9^{\mathrm{a}}$ & $3,3 \pm 8,2^{\mathrm{a}}$ \\
\hline & 15 & $83,3 \pm 8,2^{b}$ & $18,7 \pm 14,5^{b}$ \\
\hline \multicolumn{4}{|l|}{ Etilenoglicol } \\
\hline \multicolumn{2}{|l|}{ Ethylene glycol } & $90,0 \pm 0,0^{\mathrm{a}}$ & \\
\hline & 10 & $88,3 \pm 4,1^{\mathrm{a}}$ & $40,8 \pm 10,6^{\mathrm{b}}$ \\
\hline & 15 & $88,3 \pm 4,1^{\mathrm{a}}$ & $55,0 \pm 22,0^{\mathrm{c}}$ \\
\hline \multicolumn{4}{|l|}{ DMA } \\
\hline & 10 & $\begin{array}{l}76,7 \pm 24,2^{\mathrm{a}} \\
81,7 \pm 18,3^{\mathrm{a}}\end{array}$ & $\begin{array}{l}0,0 \pm 0,0^{\mathrm{a}} \\
0,0 \pm 0,0^{\mathrm{a}}\end{array}$ \\
\hline & 15 & $5,2 \pm 12,2^{b}$ & $0,0 \pm 0,0^{\mathrm{a}}$ \\
\hline \multicolumn{4}{|l|}{$\begin{array}{l}\text { Propanodiol } \\
\text { Propanediol }\end{array}$} \\
\hline & 5 & $88,3 \pm 9,8^{\mathrm{a}}$ & $0.0 \pm 0.0^{\mathrm{a}}$ \\
\hline & 10 & $90,0 \pm 0,0^{\mathrm{a}}$ & $3,7 \pm 9,0^{\mathrm{a}}$ \\
\hline & 15 & $90,0 \pm 0,0^{\mathrm{a}}$ & $7,8 \pm 12,2^{\mathrm{a}}$ \\
\hline
\end{tabular}

DMSO = dimetilsulfóxido; $\mathrm{DMA}=$ dimetilacetamida .

a-c Médias de um mesmo crioprotetor cujo superscrito contém uma letra igual são semelhantes $(p<0,05)$.

$D M S O=$ dimethylsulfoxide; $D M A=$ dimethylacetamide .

a-c Means in the same cryoprotectant whose superscript has a same letter are similar $(p<.05)$.

(interno e externo) são combinados, por exemplo, metanol e leite-em-pó ou DMSO e gema de ovo (Harvey, 1983). Gema de ovo (Babiak et al., 1995) e leite em pó (Bart et al., 1998) têm sido utilizados com sucesso para sêmen de peixes. O leite em pó elevou a taxa de motilidade pós-descongelamento quando combinado com metanol em Sarotherodon mossambicus (Harvey, 1983). O metanol, quando associado à gema de ovo, não produziu resultados satisfatórios em Leporinus macrocephalus (Ribeiro, 2001), em razão de aparentemente não ter ocorrido sinergismo entre esses crioprotetores (Harvey, 1983).

As taxas de motilidade espermática pós-descongelamento do sêmen criopreservado em diferentes crioprotetores encontram-se na Tabela 2. As várias soluções ativadoras não influenciaram as taxas de motilidade do sêmen criopreservado nos diferentes crioprotetores. Metanol e DMSO produziram taxas de motilidade espermática similares. Essas taxas, no entanto, foram significativamente diferentes entre metanol e etilenoglicol quando ativados com $\mathrm{NaHCO}_{3}$ $119 \mathrm{mM}$ e água destilada. O mesmo ocorreu com DMSO e etilenoglicol, quando ativados com $\mathrm{NaCl} 25 \mathrm{mM}$ e água destilada. Diferentes soluções têm sido utilizadas para ativar a motilidade espermática pósdescongelamento (água: Linhart et al., 1993; Otémé et al., 1996; soluções salinas: Tsvetkova et al., 1996).

Embora $\mathrm{NaHCO}_{3} 119 \mathrm{mM}$ seja solução ativadora amplamente preferida (Stoss \& Holtz, 1981; Yao et al., 2000), ela não se mostrou mais eficiente para o sêmen descongelado de tilápia-nilótica do que as demais empregadas no presente trabalho.

As taxas de motilidade espermática pós-descongelamento obtidas no presente trabalho foram semelhantes àquelas de Harvey \& Kelley (1988), em S. mossambicus, e mais baixas que as de Harvey (1983), também em S. mossambicus. Segundo Chao et al. (1987), motilidade espermática, em vez de taxa de fertilização, é o critério mais adequado para avaliar-se o sucesso da criopreservação do sêmen de 
tilápias. Segundo esses autores, a fertilização artificial em tilápias ainda carece de indicações ("pistas") mais seguras acerca do momento da fertilização de sorte que resultados insatisfatórios são obtidos mesmo com a utilização de sêmen fresco.

O efeito das diferentes diluições do sêmen criopreservado com metanol (Tabela 3) ou DMSO (Tabela 4) sobre as taxas de motilidade espermática pós-descongelamento não foi significativo $(\mathrm{P}>0,05)$. Do mesmo modo, a duração da motilidade espermática do sêmen criopreservado com metanol em diferentes diluições não se alterou significativamente $(\mathrm{P}>0,05)$ (Tabela 3). Todavia, em relação ao DMSO, a diluição de 1:3 permitiu duração significativamente mais alta da motilidade espermática $(\mathrm{P}<0,05)$ (Tabela 4). Taxas de diluição do sêmen entre $1: 1$ e $1: 3$ têm sido rotineiramente empregadas em várias espécies de clima de temperado (Lahnsteiner, 2000; Cloud, 2000). Taxas de diluição mais altas têm sido utilizadas para tilápias com resultados satisfatórios (1:5 em S. mossambicus: Harvey, 1983; 1:9 em $O$. mossambicus: Harvey \& Kelley, 1988). Chao et al. (1987) obtiveram ótima motilidade espermática com a diluição de 1:1 para Oreochromis spp, Tilapia zillii e híbridos, enquanto Rana \& McAndrew (1989) informaram que a taxa de fertilização não foi afetada quando as proporções sêmen:diluente variaram de 1:1 a 1:20 em O. niloticus, $O$. aureus e $O$. mossambicus.

As taxas de congelamento e descongelamento são variáveis críticas em criopreservação de sêmen de peixes (Viveiros et al., 2000) embora poucos trabalhos têm sido feitos em relação ao tema (Rana, 1995). Taxas adequadas de congelamento não podem ser generalizadas pois diferentes diluentes, tamanhos de palhetas e temperaturas têm sido usadas com sucesso (Lahnsteiner, 2000). No presente trabalho, o sêmen foi resfriado à taxa de $30-40^{\circ} \mathrm{C} / \mathrm{min}$ no botijão de vapor de nitrogênio líquido (dados não publicados). Taxas de congelamento ótimas foram obtidas a $30^{\circ} \mathrm{C} / \mathrm{min}$ (Harvey, 1983), entre 5 e $50^{\circ} \mathrm{C} / \mathrm{min}$ (Rana \& McAndrew, 1989) e entre 10 e $80^{\circ} \mathrm{C} / \mathrm{min}$ (Harvey \& Kelley, 1988) para o sêmen de tilápias.

Tabela 2 - Taxa de motilidade (\%) pós-descongelamento do sêmen de tilápia-nilótica (Oreochromis niloticus), var. Chitralada, diluído 1:9 em diferentes soluções diluentes e ativado em diferentes soluções ativadoras

Table 2 - Motility rate (\%) of frozen/thawed sperm of Nile tilapia (Oreochromis niloticus), var. Chitralada, diluted to 1:9 in different diluents and activated in different activating solutions

\begin{tabular}{|c|c|c|c|}
\hline \multirow[t]{2}{*}{$\begin{array}{l}\text { Solução ativadora } \\
\text { Activating solution }\end{array}$} & \multicolumn{3}{|c|}{$\begin{array}{l}\text { Crioprotetor }(\text { concentração final }=9 \%) \\
\text { Cryoprotector }(\text { final concentration }=9 \%)\end{array}$} \\
\hline & $\begin{array}{l}\text { Metanol } \\
\text { Methanol }\end{array}$ & $\begin{array}{l}\mathrm{DMSO} \\
D M S O\end{array}$ & $\begin{array}{l}\text { Etilenoglicol } \\
\text { Ethylene glycol }\end{array}$ \\
\hline $\begin{array}{l}\mathrm{NaHCO}_{3} 119 \mathrm{mM} \\
\mathrm{NaCl} 25 \mathrm{mM} \\
\text { Água destilada } \\
\text { Distilled water }\end{array}$ & $\begin{array}{l}28,3 \pm 27,9^{\mathrm{aA}} \\
16,4 \pm 20,1^{\mathrm{aAB}} \\
25,0 \pm 28,1^{\mathrm{aA}}\end{array}$ & $\begin{array}{l}33,5 \pm 26,1^{\mathrm{aAB}} \\
35,0 \pm 12,9^{\mathrm{aA}} \\
35,0 \pm 18,7^{\mathrm{aA}}\end{array}$ & $\begin{array}{l}5,8 \pm 5,0^{\mathrm{aB}} \\
5,1 \pm 4,9^{\mathrm{aB}} \\
4,8 \pm 5,0^{\mathrm{aB}}\end{array}$ \\
\hline \multicolumn{4}{|c|}{$\begin{array}{l}\text { a-b Médias nas colunas com sobrescritos contendo letras minúsculas diferentes são significativam } \\
\text { A-B Médias nas linhas com sobrescritos contendo letras maiúsculas diferentes são significativamen } \\
\text { a-b Means within the same column followed by different lowercase superscript are significantly different }(p>\text {.05). } \\
\text { A-B Means within the same line followed by different uppercase superscript are not significantly different }(p>.05) \text {. }\end{array}$} \\
\hline \multicolumn{4}{|c|}{$\begin{array}{l}\text { Tabela } 3 \text { - Taxa e duração da motilidade espermática (média } \pm \text { desvio-padrão) do sêmen de tilápia-nilótica (Oreochrom } \\
\text { niloticus), var. Chitralada, congelado em diferentes taxas de diluição e concentrações de metanol } \\
\text { Table } 3 \text { - Motility rate and lenght (mean } \pm \text { standard deviation) of Nile tilapia (Oreochromis niloticus), var. Chitralada, sperm froze } \\
\text { in different dilution rates and in different methanol concentrations }\end{array}$} \\
\hline Taxa de diluição & Concentração final do crioprotetor $(\%)$ & Taxa de motilidade $(\%)$ & Duração da motilidade \\
\hline Dilution rate & Cryoprotector final concentration & Motility rate & Motilitylenght \\
\hline $1: 1$ & 7,5 & $31,3 \pm 21,7^{\mathrm{a}}$ & $110,9 \pm 103,1^{\mathrm{a}}$ \\
\hline $1: 2$ & 10,0 & $46,3 \pm 15,1^{\mathrm{a}}$ & $203,8 \pm 99,3^{\mathrm{a}}$ \\
\hline $1: 3$ & 11,3 & $43,3 \pm 15,1^{\mathrm{a}}$ & $241,2 \pm 57,3^{\mathrm{a}}$ \\
\hline $1: 4$ & 12,0 & $35,0 \pm 17,7^{\mathrm{a}}$ & $155,3 \pm 131,8^{\mathrm{a}}$ \\
\hline
\end{tabular}

${ }^{a}$ Médias com mesmo sobrescrito são similares $(P>0,05)$.

${ }^{a}$ Means followed by the same superscript are similar $(P>.05)$. 
Tabela 4 - Taxa e duração da motilidade espermática (média \pm desvio-padrão) do sêmen de tilápia-nilótica (Oreochromis niloticus), var. Chitralada, congelado em diferentes taxas de diluição e concentrações de DMSO

Table 4 - Motility rate and lenght (mean \pm standard deviation) of Nile tilapia (Oreochromis niloticus), var. Chitralada sperm frozen in different dilution rates and in different concentrations of DMSO

\begin{tabular}{lccc}
\hline $\begin{array}{l}\text { Taxa de diluição } \\
\text { Dilution rate }\end{array}$ & $\begin{array}{c}\text { Concentração final do crioprotetor (\%) } \\
\text { Cryoprotector final concentration }\end{array}$ & $\begin{array}{c}\text { Taxa de motilidade (\%) } \\
\text { Motilityrate }\end{array}$ & $\begin{array}{c}\text { Duração da motilidade } \\
\text { Motility lenght }\end{array}$ \\
\hline $1: 1$ & 5,0 & $35,0 \pm 19,3^{\mathrm{a}}$ & $96,8 \pm 92,3^{\mathrm{b}}$ \\
$1: 2$ & 6,7 & $30,0 \pm 18,5^{\mathrm{a}}$ & $78,6 \pm 53,8^{\mathrm{c}}$ \\
$1: 3$ & 7,5 & $35,0 \pm 5,5^{\mathrm{a}}$ & $225,2 \pm 63,9^{\mathrm{a}}$ \\
$1: 4$ & 8,0 & $20,6 \pm 12,1^{\mathrm{a}}$ & $50,0 \pm 69,6^{\mathrm{c}}$ \\
\hline
\end{tabular}

a-c Médias com mesmo sobrescrito são similares $(P>0,05)$.

a-c Means followed by the same superscript are similar $(P>.05)$.

\section{Conclusões}

A técnica de congelamento de sêmen de tilápianilótica, var. Chitralada, utilizando-se o botijão de vapor de nitrogênio líquido como recipiente criogênico, é utilizada pela primeira vez em tilápia-nilótica, Oreochromis niloticus. O emprego desse botijão nos trabalhos de campo foi facilitada em razão de sua portabilidade, além de originar resultados semelhantes àqueles obtidos por métodos tradicionais de congelamento. Os diluentes feitos pela combinação de DMSO com gema de ovo e de metanol com leite em pó, associados à glicose e água destilada, mostraram-se igualmente adequados para o congelamento do sêmen dessa espécie. As proporções de diluente: sêmen de 1:1-1:4 e 1:9 foram também satisfatórias. Soluções de NaHCO3 119 mM, NaCl 25 mM e água destilada podem ser utilizadas, com igual sucesso, na ativação da motilidade espermática do sêmen descongelado.

\section{Agradecimento}

Ao Fundo de Incentivo à Pesquisa (FIP) e à PUC Minas, pelo auxílio financeiro concedido. À Multi Gene Aqüicultura, Fazenda Água Fria, C.P. 46, Esmeraldas, MG, pelas facilidades concedidas no uso de suas instalações e no fornecimento dos peixes. À A.T.M. Viveiros, pelas valiosas críticas e sugestões ao manuscrito.

\section{Literatura Citada}

BABIAK, I.; GLOGOWSKI, J.; LUCZYNSKI, M.J. et al. Cryopreservation of the milt of the Northern pike. Journal of Fish Biology, v.46, p.819-828, 1995.

BART, A.N.; WOLFE, D.F.; DUNHAM, R.A. Cryopreservation of blue catfish spermatozoa and subsequent fertilization of channel catfish eggs. Transactions of the American Fisheries Society, v.127, n.5, p.819-824, 1998.
BOSCOLO, W.R.; HAYASHI, C.; SOARES, C.M. et al. Desempenho e características de carcaça de machos revertidos de tilápias do Nilo (Oreochromis niloticus), linhagens tailandesa e comum, nas fases inicial e de crescimento. Revista Brasileira de Zootecnia, v.30, n.5, p.1391-1396, 2001.

CHAO, N-H.; CHAO, W-C.; LIU, K-C. et al. The properties of tilapia sperm and its cryopreservation. Journal of Fish Biology, v.30, p.107-118, 1987.

CLOUD, J.G. Cryopreservation of sperm of steelhead rainbow trout after refrigerated storage. In: TIERSCH, T.R.; MAZIK, P.M. (Eds.) Cryopreservation in aquatic species. Baton Rouge, Louisiana: World Aquaculture Society, 2000. p.101-103.

ENGLE, C.R. Economics of tilapia aquaculture. In: COSTAPIERCE, B.A.; RAKOCY, J.E. (Eds.). Tilapia aquaculture in the Americas. Baton Rouge: The World Aquaculture Society, 1997. v.1, p.229-243.

FAO Fisheries Circular nº 886, Ver. 1, Roma, FAO, 1997, 163p. Disponível em : http://www.fao.org. Citado: 22 de agosto de 2002, ISSN 0429-9329.

HARVEY, B. Cryopreservation of Sarotherodon mossambicus spermatozoa. Aquaculture, v.32, p.313-320, 1983.

HARVEY, B. An overview of action before extinction. In: HARVEY, B.; ROSS, C.; GREER, D. et al. (Eds.). Action before extinction: an international conference on conservation of fish genetic diversity. Vancouver: World Fisheries Trust, 1998. p.1-18.

HARVEY, B. The application of cryopreservation in fish genetic conservation in North and South America. In: TIERSCH, T.R.; MAZIK, P.M. (Eds.). Cryopreservation in aquatic species. Baton Rouge, Louisiana: World Aquaculture Society, 2000. p.332-337.

HARVEY, B.; CAROLSFELD, J. Induced breeding in tropical fish culture. Otawa: International Development Research Center, 1993. 144p.

HARVEY, B.J.; KELLEY, R.N. Practical methods for chilled and frozen storage of tilapia spermatozoa. In: INTERNACIONAL SYMPOSIUM ON TILAPIA IN AQUACULTURE, 2., 1988, Bangkok, Manila. Proceedings... Bangkok: Departament of Fisheries and International Center for Living Aquatic Resources Management, 1988. p.623.

HORVÁTH, Á.; URBÁNYI, B. The effect of cryoprotectants on the motility and fertilizing capacity of cryopreserved African catfish Clarias gariepinus (Burchell 1822) sperm. Aquaculture Research, v.31, p.317-324, 2000.

LAHNSTEINER, F. Semen cryopreservation in the Salmonidae and in the Northern pike. Aquaculture Research, v.31, p.245-258, 2000.

LEUNG, L.K.P. Principles of biological cryopreservation. In: 
JAMIESON, B.G.M. (Ed.) Fish evolution and systematics: evidence from spermatozoa. Cambridge: Cambridge University Press, 1991. p.231-244.

LINHART, O.; BILlARD, R.; PROTEAU, J.P. Cryopreservation of European catfish (Silurus glanis L.) spermatozoa. Aquaculture, v.115, p.347-359, 1993.

MONGKONPUNYA, K.; PUPIPAT, T.; TIERSCH, T.R. Cryopreservation of sperm of Asian catfishes including the endangered Mekong giant catfish. In: TIERSCH, T.R.; MAZIK, P.M. (Eds.). Cryopreservation in aquatic species. Baton Rouge, Louisiana: World Aquaculture Society, 2000. p.108-116.

OTÉMÉ, Z.J.; NUNEZ RODRIGUES, J.; KOUASSI, C.K. et al. Testicular structure, spermatogenesis and sperm cryopreservation in the African clariid catfish Heterobranchus longifilis (Valenciennes, 1840). Aquaculture Research, v.27, p.805-813, 1996.

PIIRONEN, J. Cryopreservation of sperm from brown trout (Salmo trutta m. lacustris L.) and arctic charr (Salvelinus alpinus L.). Aquaculture, v.116, p.275-285, 1993.

RANA, K. Preservation of gametes. In: BROMAGE, N.R.; ROBERTS, R.J. (Eds.). Broodstock management and egg and larval quality. London: Blackwell Science, 1995. p.53-75.

RANA, K.J.; McANDREW, B.J. The viability of cryopreserved tilapia spermatozoa. Aquaculture, v.76, p.335-345, 1989.

RIBEIRO, R.I.M.A. Criopreservação do sêmen de piau-açu Leporinus macrocephalus (Garavello \& Britski, 1998). Belo Horizonte: Universidade Federal de Minas Gerais, 2001. 65p. Dissertação (Mestrado em Biologia Celular) Universidade Federal de Minas Gerais, 2001.
STOSS, J.; HOLTZ, W. Cryopreservation of rainbow trout (Salmo gairdneri) sperm I. Effect of thawing solution, sperm density and interval between thawing and insemination. Aquaculture, v.22, p.97-104, 1981.

TSVETKOVA, L.I.; COSSON, J.; LINHART, O. et al. Motility and fertilizing capacity of fresh and frozen-thawed spermatozoa in sturgeons Acipenser baeri and A. ruthenus. Journal of Applied Ichthyology, v.12, p.107-112, 1996.

VIVEIROS, A.T.M.; SO, N.; KOMEN, J. Sperm cryopreservation of African catfish, Clarias gariepinus: cryoprotectants, freezing rates and sperm:egg dilution ratio. Theriogenology, v.54, p.1395-1408, 2000.

WAYMAN, W.R.; TIERSCH, T.R. Research methods for cryopreservation of sperm. In: TIERSCH, T.R.; MAZIK, P.M. (Eds.). Cryopreservation in aquatic species. Baton Rouge, Louisiana: World Aquaculture Society, 2000. p.264-275.

YAO, Z.; CRIM, L.W.; RICHARDSON, G.P. et al. Motility, fertility and ultrastructural changes of ocean pout (Macrozoarces americanus L.) sperm after cryopreservation. Aquaculture, v.181, p.361-375, 2000.
Recebido em: 04/10/02

Aceito em: 03/02/03 